\title{
Herzinsuffiziente profitieren von i.v. Eisentherapie
}

\begin{abstract}
Eisen ist für die Blutbildung unverzichtbar. Doch unabhängig von dem Vorliegen einer Anämie lässt sich bei Patienten mit chronischer Herzinsuffizienz durch eine i.v. Eisentherapie die kardiale Symptomatik signifikant verbessern, so das Ergebnis der FAIR-HF-(Ferinject ${ }^{\circledR}$ Assessment in patients with Iron deficiency and chronic Heart Failure-)Studie.
\end{abstract}

— Dabei handelt es sich um eine große multizentrische, randomisierte, doppelblinde, placebokontrollierte PhaseIII-Studie bei Patienten mit chronischer Herzinsuffizienz und Eisenmangel mit oder ohne Anämie. Im Rahmen dieser Studie wurde untersucht, ob die Korrektur des Eisenmangels mit intravenösem Eisen (Ferric Carboxymaltose) die Symptomatik betroffener Patienten günstig beeinflusst. Einbezogen in die Studie wurden 459 chronisch herzinsuffiziente Patienten mit Eisenmangel, die placebokontrolliert, randomisiert Ferinject ${ }^{\circledR}$ erhielten, und zwar zunächst wöchentlich, bis der Eisenmangel ausgeglichen war, und dann noch monatlich bis zur 24 . Woche im Sinne einer Erhaltungstherapie.

Das Studienergebnis war signifikant: Die mit Eisen behandelten Patienten zeigten eine deutliche Verbesserung sowohl der Lebensqualität als auch der NYHA-Klasse. In der mit

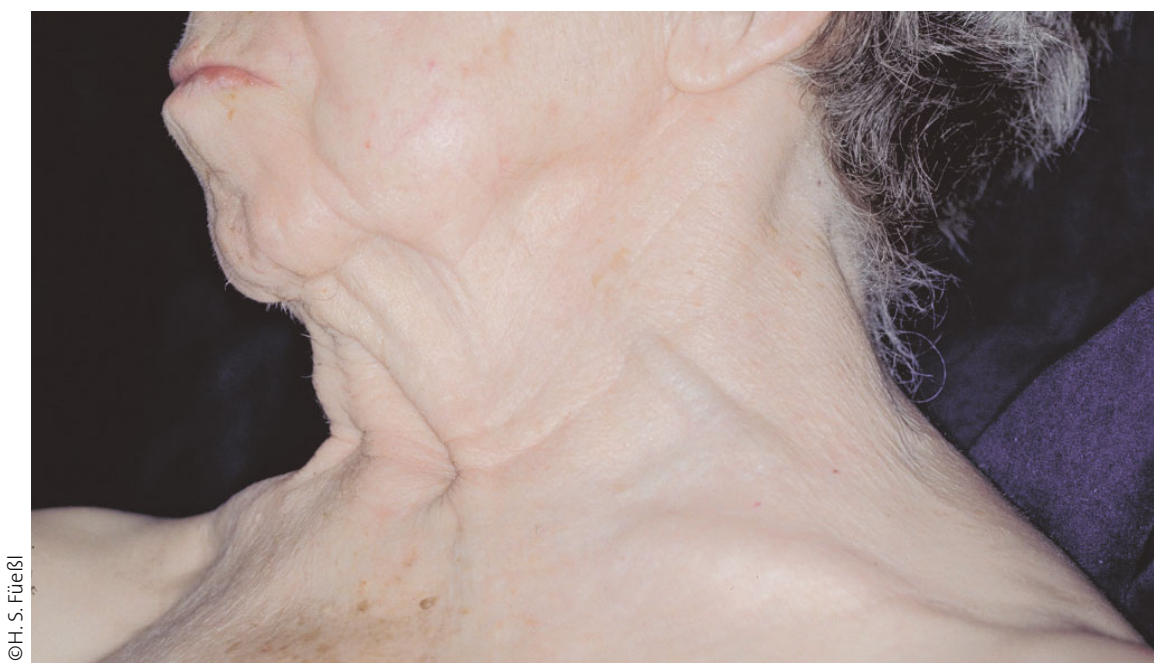

Einflussstauung bei Herzinsuffizienz.

dem Verum behandelten Patientengruppe gaben in der 24. Studienwoche 50\% der Patienten eine deutliche Verbesserung ihrer Leistungsfähigkeit an im Vergleich zu nur 28\% der Patienten in der Placebogruppe. Im Hinblick auf die NYHA-Klassifikation befanden sich $47 \%$ der mit Ferinject ${ }^{\circledR}$ behandelten Patienten in der 24. Woche in der NYHA-Klasse I oder II gegenüber nur 30\% der Patienten, die Placebo erhielten.

Durch die Eisentherapie verlängerte sich die Gehstrecke im 6-MinutenGehtest nach 24 Wochen um 39 Meter im Vergleich zu neun Metern in der Placebogruppe. Bereits ab der vierten Studienwoche schätzten die mit dem Eisenpräparat behandelten Patienten ihre Lebensqualität signifikant besser ein als die Placebogruppe. Die Sterblichkeitsrate und die Häufigkeit unerwünschter Nebenwirkungen einschließlich der Hospitalisierung waren in beiden Patientengruppen gleich.

\section{Kommentar}

Bisher wurde im klinischen Alltag einem Eisenmangel, wenn er nicht zu einer signifikanten Anämie geführt hatte und wenn keine eindeutige Ursache im Sinne einer Blutungsquelle gefunden wurde, keine besondere Bedeutung beigemessen. Doch diese Studie zeigt nun erstmals, dass ein Eisenmangel bei herzinsuffizienten Patienten unabhängig von dem Vorliegen einer Anämie für die Symptomatik und somit die Lebensqualität von besonderer Bedeutung ist. Daraus ergibt sich als Konsequenz für den praktischen Alltag, dass bei jedem herzinsuffizienten Patienten zunächst Ferritin bestimmt werden sollte. Liegt der Wert unter $100 \mu \mathrm{g} / \mathrm{l}$, so ist damit ein Eisenmangel bewiesen. Bei Ferritinwerten zwischen 100 und $299 \mu \mathrm{g} / \mathrm{l}$ sollte zunächst die Transferinsättigung bestimmt werden. Liegt dieser Wert < $20 \%$, muss man ebenfalls von einem Eisenmangel ausgehen, der nach diesen überzeugenden Studienergebnissen immer mit einem intravenösen Eisenpräparat behandelt werden sollte.

P. Stiefelhagen .

\section{Promotion / Habilitation. Finden der passenden Professur. www.promotion-d.de}

Tel.: 0681 / 7097 689, Fax 0681 / 7097691

West Promotionshilfe $\mathrm{GmbH}$
S. D. Anker et al.

Ferric carboxymaltose in patients with heart failure and iron deficiency. 10.1056/NEJMoaogo8355 